Supporting Information for the Manuscript:

\title{
Tuning the Structural and Magnetic Properties of Thermally Robust Coordination Polymers
}

Norberto Masciocchi, ${ }^{a}$ Simona Galli, ${ }^{\mathrm{a}, *}$ Angelo Sironi, ${ }^{\mathrm{b}}$ Elena Cariati, ${ }^{\mathrm{c}}$ Miguel A. Galindo, ${ }^{\mathrm{d}}$ Elisa Barea, ${ }^{\mathrm{d}} \mathrm{M}^{\mathrm{a}}$ Angustias Romero, ${ }^{\mathrm{d}}$ Juan M. Salas, ${ }^{\mathrm{d}}$ Jorge A. R. Navarro ${ }^{\mathrm{d}, *}$ and Francisco Santoyo-González ${ }^{\mathrm{d}}$

\footnotetext{
${ }^{a}$ Dipartimento di Scienze Chimiche e Ambientali, Università dell'Insubria, Via Valleggio 11, 22100 Como (Italy) Fax: +39-031-326230; e-mail: simona.galli@uninsubria.it

${ }^{b}$ Dipartimento di Chimica Strutturale e Stereochimica Inorganica, Università di Milano and Istituto di Scienze e Tecnologie Molecolari del CNR (ISTM-CNR), Via Venezian 21, 20133 Milano (Italy)

${ }^{c}$ Dipartimento di Chimica Inorganica, Metallorganica e Analitica, Università di Milano and Istituto di Scienze e Tecnologie Molecolari del CNR (ISTM-CNR), Via Venezian 21, 20133 Milano (Italy)

${ }^{d}$ Departamentos de Química Inorgánica y Orgánica, Universidad de Granada, Av. Fuentenueva S/N, 18071 Granada (Spain)-Fax: +34-958-248526; e-mail: jarn@ugr.es
} 

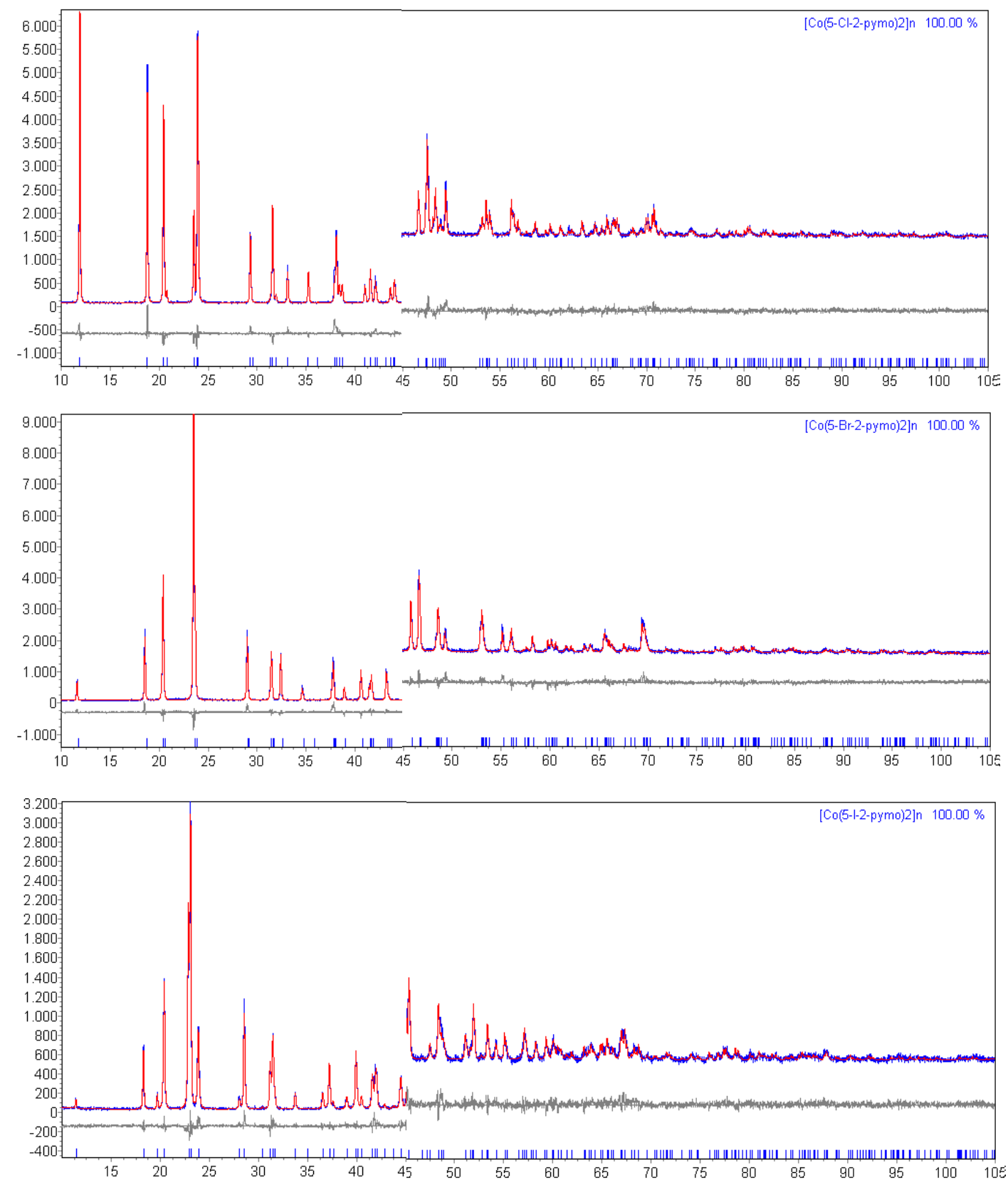

Figure S1: From top, Rietveld refinement results for $[\mathrm{Co}(5-\mathrm{Cl}-2-\text { pymo })]_{\mathrm{n}}, \mathbf{1}_{\mathbf{C l}},[\mathrm{Co}(5-\mathrm{Br}-2-\mathrm{pymo})]_{\mathrm{n}}$, $\mathbf{1}_{\mathbf{B r}}$, and [Co(5-I-2-pymo) $]_{\mathrm{n}}, \mathbf{1}_{\mathbf{I}}$, as appreciable from experimental, calculated and difference diffraction patterns. Peak markers at the bottom. For the sake of clarity, the portion above 45 deg has been magnified $(3 \times)$. Horizontal axis: $2 \theta$, deg. Vertical axis: counts. 

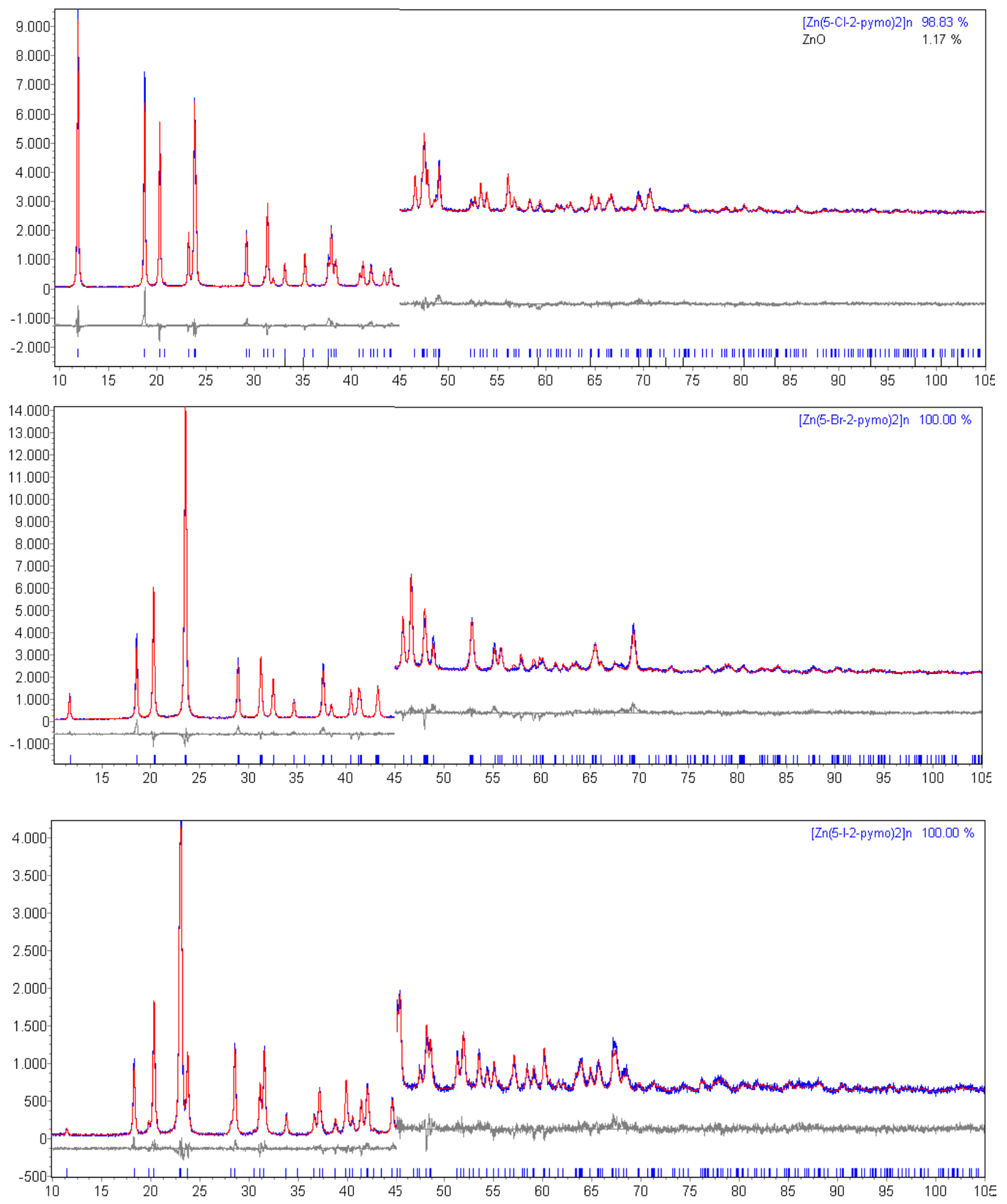

Figure S2: From top, Rietveld refinement results for $[\mathrm{Zn}(5-\mathrm{Cl}-2-\mathrm{pymo})]_{\mathrm{n}}, \mathbf{2}_{\mathrm{Cl}},[\mathrm{Zn}(5-\mathrm{Br}-2-\mathrm{pymo})]_{\mathrm{n}}$, $\mathbf{2}_{\mathbf{B r}}$, and $[\mathrm{Zn}(5-\mathrm{I}-2-\mathrm{pymo})]_{\mathrm{n}}, \mathbf{2}_{\mathrm{I}}$, as appreciable from experimental, calculated and difference diffraction patterns. Peak markers at the bottom. For the sake of clarity, the portion above 45 deg has been magnified $(3 \times)$. Horizontal axis: $2 \theta$, deg. Vertical axis: counts. 